\title{
Data-Driven Chance-Constrained Design of Voltage Droop Control for Distribution Networks
}

\author{
Joshua Comden Ahmed S. Zamzam Andrey Bernstein \\ National Renewable Energy Laboratory \\ \{joshua.comden, ahmed.zamzam, andrey.bernstein\}@nrel.gov
}

\begin{abstract}
This paper addresses the design of local control methods for voltage control in distribution networks with high levels of distributed energy resources (DERs). The designed control methods modulate the active and reactive power output of DERs proportional to the deviation of the local measured voltage magnitudes from a reference voltage, which is referred to as droop control; thus, the design focuses on determining the droop characteristics that satisfy network-wide voltage magnitude constraints. The uncertainty and variability of DERs renders the design of optimal droop controls very challenging; hence, this paper proposes chance constraints to limit the risk from intermittent DERs by designing droop control coefficients that guarantee the satisfaction of network operational constraints with a specific probability. In addition, the proposed approach relies entirely on historical data rather than assuming knowledge of the probability distributions that characterize the uncertainty of DERs. The efficacy of the proposed method is demonstrated on a 37-bus distribution feeder.
\end{abstract}

\section{Introduction}

As volatile renewable energy increases its share of the energy resource mix, power system operational and control objectives become more challenging. For example, distributed energy resources (DERs) at the edge of a distribution network can cause voltage violations, especially when the generation exceeds the load on a feeder [1]. On the other hand, DERs have the potential to solve or mitigate their own issues through different control technologies (e.g., smart inverters combined with local storage) and from the fact that they are located at various locations on a distribution network [2].

When it comes to making sure that voltage magnitudes stay within their prescribed bounds, a standard technique called voltage droop control has
DERs adjust their individual reactive power injections in proportion to locally measured voltage magnitudes [3]. Although at a local level this is a very simple and easy to implement control law, $[4,5]$ showed that the actual droop control settings should be based on their location in the distribution network; otherwise, instability can occur.

A natural goal for global droop control design is to implement robust settings guaranteeing that, under any situation, the voltages everywhere stay within their bounds [6]; however, this might not be practical or justifiable for many reasons. There might not be enough capacity among the available controllers to satisfy the guarantees, but adding any additional needed capacity could be prohibitively expensive [7]. On the other hand, limiting some uncertainty in the system might require curtailing or removing the most volatile resources, such as wind and solar, which goes against many future objectives for having a clean energy grid [8]; thus, the key to this balance is how uncertainty is framed and handled.

In stochastic optimization, where uncertainty is modeled as a probability distribution of random variables, a common relaxation from a robust constraint that must be satisfied under all circumstances is a chance constraint that allows the violation of a bound under a limited probability [9]. In the case of voltage droop control, this would mean allowing voltages to go outside of their prescribed bounds as long as this could happen only with a small prescribed probability. Although in the ideal case it might be desired to set this probability to zero, a nonzero but small probability could allow the existing control capabilities to have feasible and functional droop control settings.

Chance constraints bring their own set of challenges, however, which makes them difficult to use in general practical applications. The first is that they are not convex even if the bound being relaxed probabilistically is linear [10], except in some special cases (e.g., the probability distribution is Gaussian or uniform and symmetric [11]). The second challenge is that the 
probability distribution for the uncertainty is assumed to be known, but this is not the case in many applications. This creates the necessity to use an empirical distribution built from historical data in place of the true distribution.

In the past decade, many methods have been developed for this particular data-driven situation; however, they have the added consequence of greatly increasing the time complexity to solve an optimization problem (e.g., [12]), especially for high-dimensional data, which are often found in energy system problems. The recent work by [13] designed a simple but effective method of approximating chance constraints with data-driven, deterministic, second-order-cone constraints. They get around the time complexity problems found in other methods by first using the data to calculate the sample mean and covariance of the high-dimensional data and using those in the optimization problem. We apply this data-driven method to the application of voltage droop control.

The main contributions of this paper can be summarized as follows:

- We formulate the voltage droop control decision problem as a chance-constrained stochastic optimization problem. (Section 2)

- We decouple the chance constraints and approximate the stochastic optimization problem with a data-driven deterministic form that guarantees satisfaction of the original chance constraints. (Section 3)

- We evaluate the performance of the proposed data-driven method on an unbalanced three-phase IEEE distribution test case using real-world photovoltaic (PV) and load data. Results show that using the proposed data-driven method can better control the voltage magnitude compared to standard IEEE guidelines. (Section 4)

\subsection{Notation}

Throughout this paper, all matrices (vectors) are denoted by upper- (lower-) case boldface letters, whereas $(\cdot)^{\top}$ stands for vector or matrix transpose. The operator $\operatorname{diag}(\mathbf{x})$ is a diagonal matrix holding in order entries of vector $\mathbf{x}$ on its diagonal. The boldface one, $\mathbf{1}$ (zero $\mathbf{0}$ ), is used to denote an all-ones (zeros) matrix with the respective dimensions. The $L_{p}$-norm of a vector or a matrix is denoted by $\|\cdot\|_{p}$.

\section{Problem Formulation}

\subsection{Power System Model}

Consider a distribution network with the set of nodes $\{0\} \cup \mathcal{N}$, where node 0 denotes the substation, and $\mathcal{N}:=$ $\{1, \ldots, N\}$. Let $\left\{\mathbf{p}_{t}, \mathbf{q}_{t}\right\} \in \mathbb{R}^{N}$, respectively, be the vector of the net real and reactive power injections at each node in $\mathcal{N}$ at time $t \in\{1, \ldots, T\}$, where $T$ is the time horizon considered for the control settings or the uncontrollable power injection forecasts.

In this work, the relationship between the voltage magnitudes, denoted by $\mathbf{v}_{t} \in \mathbb{R}^{N}$, and the power injections at time $t$ is approximated using a linear power flow model:

$$
\mathbf{v}_{t}=\mathbf{R} \mathbf{p}_{t}+\mathbf{B} \mathbf{q}_{t}+\mathbf{a}
$$

where $\{\mathbf{R}, \mathbf{B}\} \in \mathbb{R}^{N \times N}$ and $\mathbf{a} \in \mathbb{R}^{N}$ can be derived from the admittance matrix (e.g., [14]) or data (e.g, [15]). Linearized power flow equations allow us to formulate a convex optimization problem that can be solved efficiently and allow the use of linear algebra techniques in the controller design. Although the theoretical development will be with linearized power flow equations, the resulting control algorithms will be evaluated with a nonlinear $\mathrm{AC}$ power flow model in Section 4 which shows that linearization error has minimal impact on the results.

Remark 1. The particular linearization method given by [14] can be derived from the block-partitioned nodal admittance matrix $\mathbf{Y}:=\left[\mathbf{Y}_{00} \mathbf{Y}_{0 L} ; \mathbf{Y}_{L 0} \mathbf{Y}_{L L}\right]$ and the nominal voltage at the substation $v_{0}$.

$$
\begin{aligned}
\mathbf{w} & :=-\mathbf{Y}_{L L}^{-1} \mathbf{Y}_{L 0} v_{0} \\
\mathbf{M} & :=\mathbf{Y}_{L L}^{-1} \operatorname{diag}(\overline{\mathbf{w}})^{-1} \\
\mathbf{R} & :=\operatorname{diag}(|\mathbf{w}|) \Re\left(\operatorname{diag}(\overline{\mathbf{w}})^{-1} \mathbf{M}\right) \\
\mathbf{B} & :=\operatorname{diag}(|\mathbf{w}|) \Re\left(\operatorname{diag}(\overline{\mathbf{w}})^{-1}(-\jmath \mathbf{M})\right) \\
\mathbf{a} & :=|\mathbf{w}|
\end{aligned}
$$

where $\mathrm{w}$ is the nodal zero-load voltages (not to be confused with the vector of random variables $\omega$ described later).

\subsection{Stochastic Load Model}

Let $\left(\widetilde{\mathbf{p}}_{t}, \widetilde{\mathbf{q}}_{t}\right)$ be the uncontrollable power injections and $(\widehat{\mathbf{p}}, \widehat{\mathbf{q}})$ be their static forecasts over the time horizon $T$. Denote the forecasting errors as the following random variables $\left(\boldsymbol{\omega}_{t}^{p}, \boldsymbol{\omega}_{t}^{q}\right)$, and hence, the 
uncontrollable power injections are given by:

$$
\begin{aligned}
\widetilde{\mathbf{p}}_{t} & =\widehat{\mathbf{p}}+\boldsymbol{\omega}_{t}^{p} \\
\widetilde{\mathbf{q}}_{t} & =\widehat{\mathbf{q}}+\boldsymbol{\omega}_{t}^{q} .
\end{aligned}
$$

In other words, $(\widehat{\mathbf{p}}, \widehat{\mathbf{q}})$ are forecasts of the average uncontrollable power injections over the time horizon $T$, and $\left(\boldsymbol{\omega}_{t}^{p}, \boldsymbol{\omega}_{t}^{q}\right)$ are the time-varying deviations from the forecasted average.

\subsection{Voltage Droop Control}

Voltage droop controls operate in a grid-following mode by adjusting power injections proportionally to a measured voltage magnitude difference from a reference, $\mathbf{v}_{\text {ref }} \in \mathbb{R}^{N}$. Let $\left\{\mathbf{G}_{p}, \mathbf{G}_{q}\right\} \in \mathbb{R}_{+}^{N \times N}$ be the diagonal matrices of the voltage droop coefficient vectors $\left\{\mathbf{g}_{p}, \mathbf{g}_{q}\right\} \in \mathbb{R}_{+}^{N}$ so that voltage droop-controlled power injections at time $t$ become:

$$
\begin{aligned}
& \mathbf{p}_{t}=\widetilde{\mathbf{p}}_{t}-\mathbf{G}_{p}\left(\mathbf{v}_{t-1}-\mathbf{v}_{\mathrm{ref}}\right) \\
& \mathbf{q}_{t}=\widetilde{\mathbf{q}}_{t}-\mathbf{G}_{q}\left(\mathbf{v}_{t-1}-\mathbf{v}_{\mathrm{ref}}\right) .
\end{aligned}
$$

Let the function $f\left(\mathbf{g}_{p}, \mathbf{g}_{q}\right)$ denote the system's operational cost associated with the droop control settings, which can be used to promote different droop control objectives. For example, $\left\|\left[\mathbf{g}_{p} \mathbf{g}_{q}\right]^{\top}\right\|_{2,1}$ proportionally relates the settings to the sum of absolute apparent power by summing the Euclidean norms of $\left[g_{p, i} g_{q, i}\right]^{\top}$ among buses $i \in\{1, \ldots, N\}$. This promotes that the nonzero droop controller settings will be at only a few key buses. On the other hand, $\left\|\left[\begin{array}{ll}\mathbf{g}_{p} & \mathbf{g}_{q}\end{array}\right]^{\top}\right\|_{\mathrm{F}}^{2}$ penalizes the summation droop control coefficients squares across the power network system, hence often leading to wider deployment of droop controls.

\subsection{Optimal Voltage Droop Control Problem}

The main goal of voltage droop control is to keep the voltage magnitude, $\mathbf{v}_{t}$, between the lower bound, $\underline{\mathbf{v}}$, and upper bound, $\overline{\mathbf{v}}$. Depending on the capacity of the controls available, however, it might not be possible to keep the voltage magnitude within these bounds; thus, we can soften these bounds by using the slack variable $\gamma \geq 0$ to allow for some voltage magnitudes outside the bounds:

$$
\underline{\mathbf{v}}-\gamma \mathbf{1} \leq \mathbf{v}_{t} \leq \overline{\mathbf{v}}+\gamma \mathbf{1}
$$

and then penalize any positive $\gamma$.

Because the uncontrollable power injection forecast errors $\left(\boldsymbol{\omega}_{t}^{p}, \boldsymbol{\omega}_{t}^{q}\right)$ are random variables, the above bounds with droop controls (3) are stochastic. Depending on the support of the probability distribution, the voltage bounds with the slack variable, $\gamma$, will focus only on controlling for the worst-case voltage magnitude deviations. A common way to loosen the focus of the bounds is to turn them into chance constraints that allow a specified probability of violation, $\alpha$, at each node:

$$
\begin{aligned}
\operatorname{Pr}\left(\underline{v}_{i}-\gamma \leq v_{t, i} \leq \bar{v}_{i}+\gamma\right) \geq 1-\alpha, & \\
& \forall i \in\{1, \ldots, N\} .
\end{aligned}
$$

Putting together the cost of the droop control settings, system equations (1), (2), and (3), and the chance-constrained voltage magnitude bounds (4) over the time horizon, $T$, we have the following optimal droop control problem, which depends on the probability distribution of the forecast errors $\left(\boldsymbol{\omega}_{1}^{p}, \boldsymbol{\omega}_{1}^{q}\right), \ldots,\left(\boldsymbol{\omega}_{T}^{p}, \boldsymbol{\omega}_{T}^{q}\right)$ :

$$
\begin{aligned}
\min _{\mathbf{g}_{p}, \mathbf{g}_{q}, \gamma} & f\left(\mathbf{g}_{p}, \mathbf{g}_{q}\right)+c_{\alpha} \gamma \\
\text { s.t. } & \text { (1), (2), (3) } \forall t \in\{1, \ldots, T\} \\
& \text { (4) } \forall t \in\{1, \ldots, T\} \\
& \mathbf{g}_{p} \geq \mathbf{0}, \mathbf{g}_{q} \geq \mathbf{0}, \gamma \geq 0
\end{aligned}
$$

where $c_{\alpha}$ is the per-unit cost of the voltage violation with probability $1-\alpha$, and $\mathbf{v}_{0}$ is given.

\section{Chance Constraints from Data}

The main challenges associated with solving problem (5) are: 1) the chance constraints are time-coupled through the droop control actions (3), and 2 ) the chance constraints require prior knowledge of the forecast error probability distribution. First, we modify problem (5) to decouple the chance constraints with negligible deviation from the original problem. Afterward, we use a recently developed data-driven method that can conservatively approximate the chance constraints with convex deterministic constraints built from historical data of the forecast errors.

To make the analysis more concise, we define the voltage magnitude deviation from $\mathbf{v}_{\text {ref }}$ as $\mathbf{y}_{t}:=\mathbf{v}_{t}-\mathbf{v}_{\text {ref }}$. Similarly, we define the upper and lower bounds on $\mathbf{y}$ as $\underline{\mathbf{y}}:=\underline{\mathbf{v}}-\mathbf{v}_{\text {ref }}$ and $\overline{\mathbf{y}}:=\overline{\mathbf{v}}-\mathbf{v}_{\text {ref }}$, and we also define the zero-load voltage magnitude deviation as $\mathbf{y}_{\text {ref }}:=\mathbf{a}-$ $\mathbf{v}_{\text {ref. }}$. Throughout, we will use the following definition of $\widetilde{y}_{t}$ :

$$
\widetilde{\mathbf{y}}_{t}:=\mathbf{R} \widetilde{\mathbf{p}}_{t}+\mathbf{B} \widetilde{\mathbf{q}}_{t}+\mathbf{y}_{\text {ref }}=\left[\begin{array}{lll}
\mathbf{I} & \mathbf{R} & \mathbf{B}
\end{array}\right]\left[\begin{array}{c}
\mathbf{y}_{\text {ref }} \\
\widetilde{\mathbf{p}}_{t} \\
\widetilde{\mathbf{q}}_{t}
\end{array}\right]
$$

as a compact form that represents the hypothetically uncontrolled voltage magnitude deviation. 


\subsection{Time-Decoupling of Chance-Constraints}

The method employed to decouple the voltage magnitudes in time comes from [4], which was used to derive a robust optimization problem. Inserting the droop controls (3) into the power flow equations (1) and expanding out one step of recursion gives the following:

$$
\begin{aligned}
\mathbf{y}_{t}= & \mathbf{R}\left(\widetilde{\mathbf{p}}_{t}-\mathbf{G}_{p} \mathbf{y}_{t-1}\right)+\mathbf{B}\left(\widetilde{\mathbf{q}}_{t}-\mathbf{G}_{q} \mathbf{y}_{t-1}\right)+\mathbf{y}_{\mathrm{ref}} \\
= & \widetilde{\mathbf{y}}_{t}-\left(\mathbf{R G}_{p}+\mathbf{B G}_{q}\right) \mathbf{y}_{t-1} \\
= & \widetilde{\mathbf{y}}_{t}-\left(\mathbf{R G}_{p}+\mathbf{B G}_{q}\right) \widetilde{\mathbf{y}}_{t-1} \\
& +\left(\mathbf{R G} \mathbf{G}_{p}+\mathbf{B G}_{q}\right)^{2} \mathbf{y}_{t-2} \\
= & \left(\mathbf{I}-\mathbf{R} \mathbf{G}_{p}-\mathbf{B G}_{q}\right) \widetilde{\mathbf{y}}_{t}+\left(\mathbf{R} \mathbf{G}_{p}+\mathbf{B} \mathbf{G}_{q}\right) \\
& \left(\mathbf{R}\left(\widetilde{\mathbf{p}}_{t}-\widetilde{\mathbf{p}}_{t-1}\right)+\mathbf{B}\left(\widetilde{\mathbf{q}}_{t}-\widetilde{\mathbf{q}}_{t-1}\right)\right) \\
& +\left(\mathbf{R} \mathbf{G}_{p}+\mathbf{B G}_{q}\right)^{2} \mathbf{y}_{t-2} .
\end{aligned}
$$

At this point, all the time coupling is segregated to the last two terms. Instead of using $\mathbf{y}_{t}$ directly to design the optimal droop control settings, we use the following surrogate:

$$
\begin{aligned}
\boldsymbol{\psi}_{t} & :=\left(\mathbf{I}-\mathbf{R G}_{p}-\mathbf{B G}_{q}\right) \widetilde{\mathbf{y}}_{t} \\
& =\left[\begin{array}{lll}
\mathbf{I} & -\mathbf{R} & -\mathbf{B}
\end{array}\right] \operatorname{diag}\left(\left[\begin{array}{c}
\mathbf{I} \\
\mathbf{I} \\
\mathbf{I}
\end{array}\right] \widetilde{\mathbf{y}}_{t}\right)\left[\begin{array}{c}
\mathbf{1} \\
\mathbf{g}_{p} \\
\mathbf{g}_{q}
\end{array}\right] .
\end{aligned}
$$

To be able to surrogate $\mathbf{y}_{t}$ with $\boldsymbol{\psi}_{t}$, we first assume that the control actions are happening fast enough compared to the evolution of the uncontrollable power injections so that $\left(\widetilde{\mathbf{p}}_{t}, \widetilde{\mathbf{q}}_{t}\right) \approx\left(\widetilde{\mathbf{p}}_{t-1}, \widetilde{\mathbf{q}}_{t-1}\right)$. This is a reasonable assumption because droop controllers typically operate at the $100-\mathrm{ms}$ (or faster) time granularity, whereas at this timescale, most DERs such as wind and solar vary slowly and the switching of discrete loads are relatively spread out. A sudden large disturbance (e.g., a default) might indicate a significant change in the system (e.g., power flow equations, probability distribution) which could be used to trigger a recalculation of the droop control coefficients. See Figure 5 in Section 4 for a relaxation of this assumption. Also, we assume that the $O\left(\left(\mathbf{R G}_{p}+\right.\right.$ $\left.\mathbf{B G}_{q}\right)^{2}$ ) is negligible compared to the other terms. To justify this assumption, let $\rho(\mathbf{X})$ be the spectral radius of $\mathbf{X}$. If we assume that the spectral radius $\rho\left(\mathbf{R G}_{p}+\mathbf{B} \mathbf{G}_{q}\right)$ is less than one, then the recursive terms are contracting as they go farther back in time, and it has the added benefit of guaranteeing the stability of the system (see [4] for details). A simple way to impose this assumption is by bounding the Frobenius norm $\left\|\left[\begin{array}{ll}\mathbf{G}_{p} & \mathbf{G}_{q}\end{array}\right]^{\top}\left[\begin{array}{ll}\mathbf{R} & \mathbf{B}\end{array}\right]\right\|_{F}<1$ because $\rho\left(\left[\begin{array}{ll}\mathbf{R} & \mathbf{B}\end{array}\right]\left[\begin{array}{ll}\mathbf{G}_{p} & \mathbf{G}_{q}\end{array}\right]^{\top}\right)=\rho\left(\left[\begin{array}{ll}\mathbf{G}_{p} & \mathbf{G}_{q}\end{array}\right]^{\top}\left[\begin{array}{ll}\mathbf{R} & \mathbf{B}\end{array}\right]\right) \leq$
$\left\|\left[\begin{array}{ll}\mathbf{G}_{p} & \mathbf{G}_{q}\end{array}\right]^{\top}\left[\begin{array}{ll}\mathbf{R} & \mathbf{B}\end{array}\right]\right\|_{F}$. Because the closer that $\rho\left(\mathbf{R G}_{p}+\mathbf{B} \mathbf{G}_{q}\right)$ is to 1 , the farther the surrogate, $\boldsymbol{\psi}_{t}$, will be from $\mathbf{y}_{t}$, and we can bound the Frobenius norm with the variable $\beta \in[0,1)$ :

$$
\left\|\left[\begin{array}{l}
\mathbf{G}_{p} \\
\mathbf{G}_{q}
\end{array}\right]\left[\begin{array}{ll}
\mathbf{R} & \mathbf{B}
\end{array}\right]\right\|_{F} \leq \beta
$$

and penalize $\beta$ in the objective function.

The main benefits of the surrogate, $\psi_{t}$, are that it is decoupled from the other time steps and it is linear with respect to the droop control coefficients. Further, we assume that the time horizon between the control setting decisions is small enough so that forecasting errors $\left(\boldsymbol{\omega}_{t}^{p}, \boldsymbol{\omega}_{t}^{q}\right): \forall t \in\{1, \ldots, T\}$ in Equation (2) are independent and identically distributed with respect to time, which allows us to treat all time steps similarly; thus, we can drop the associated index and modify problem (5) by replacing the time-dependent chance constraints (5c) with

$$
\begin{aligned}
: \operatorname{Pr}\left(\underline{y}_{i}-\gamma \leq \psi_{i} \leq \bar{y}_{i}+\gamma\right) \geq & 1-\alpha, \\
& \forall i \in\{1, \ldots, N\} .
\end{aligned}
$$

The $i$-th element in the surrogate, $\psi$, in (8) can be written as:

$$
\psi_{i}=\left[\begin{array}{c}
\mathbf{y}_{\mathrm{ref}} \\
\widehat{\mathbf{p}}+\boldsymbol{\omega}^{p} \\
\widehat{\mathbf{q}}+\boldsymbol{\omega}^{q}
\end{array}\right]^{\top} \quad \chi_{i}\left[\begin{array}{c}
\mathbf{1} \\
\mathbf{g}_{p} \\
\mathbf{g}_{q}
\end{array}\right]
$$

where:

$$
\boldsymbol{\chi}_{i}:=\left[\begin{array}{c}
\mathbf{I} \\
\mathbf{R} \\
\mathbf{B}
\end{array}\right]\left[\begin{array}{lll}
\mathbf{I} & \mathbf{I} & \mathbf{I}
\end{array}\right] \operatorname{diag}\left(\left[\begin{array}{c}
\mathbf{e}_{i} \\
-\mathbf{R}_{i}^{\top} \\
-\mathbf{B}_{i}^{\top}
\end{array}\right]\right),
$$

$\mathbf{e}_{i}$ is the $i$-th standard basis vector, and the index, $i$, on $\{\mathbf{R}, \mathbf{B}\}$ represents its $i$-th row. This structure shows that $\psi_{i}$ is the inner product of a stochastic vector and a linear function of a decision vector. With this structure, there exist several options to transform the chance constraints into data-driven deterministic constraints.

\subsection{Data-Driven Chance Constraints}

Depending on the family of probability distributions, the first two moments of the forecasting errors $\left(\boldsymbol{\omega}^{p}, \boldsymbol{\omega}^{q}\right)$ can be used to find an exact or conservative approximation of the above chance constraints. For example, if the forecasting errors are assumed to be Gaussian, then an exact deterministic convex reformulation can be used [16]. On the other 
hand, if the exact shape of the distribution is not known besides the first two moments, then a convex deterministic reformulation can be made that guarantees satisfaction of the original chance constraint under the worst possible distribution that has those first two moments [17]. This is sometimes referred to as a moment-based or Chebyshev-ambiguous [18] distributionally robust chance constraint.

In this paper, we adopt a moment-based distributionally robust chance constraint; however, instead of assuming knowledge of the true moments of the distribution of the forecasting errors, we use historical data to obtain an estimate of their mean and covariance. We build on the recent result in [13], which developed a data-driven method that considers the estimation error when using the sample mean and covariance in moment-based distributionally robust chance constraints.

Let $\boldsymbol{\mu}$ be the mean vector, $\boldsymbol{\Sigma}$ be the covariance matrix, and $(\underline{\omega}, \bar{\omega})$ be the elementwise lower- and upper-bound support vectors of the random forecast errors $\boldsymbol{\omega}:=\left[\begin{array}{ll}\left(\boldsymbol{\omega}^{p}\right)^{\top} & \left(\boldsymbol{\omega}^{q}\right)^{\top}\end{array}\right]^{\top}$. From $M$ independent and identically distributed samples, define $\widehat{\boldsymbol{\mu}}_{M}, \widehat{\boldsymbol{\Sigma}}_{M}$, and $\left(\widehat{\widehat{\boldsymbol{\omega}}}_{M}, \widehat{\overline{\boldsymbol{\omega}}}_{M}\right)$ as their sample derived estimations. Also define $\mathcal{P}(\boldsymbol{\mu}, \boldsymbol{\Sigma}, \underline{\boldsymbol{\omega}}, \overline{\boldsymbol{\omega}})$ as the family of probability distributions with the associated properties. To state the chance constraints in a distributionally robust form, we split the probability, $\alpha$, equally on both sides of the chance constraint (10) and take the worst-case probability distribution given the first two moments and support:

$$
\begin{aligned}
\inf _{\boldsymbol{\omega} \sim \mathcal{P}(\boldsymbol{\mu}, \boldsymbol{\Sigma}, \underline{\boldsymbol{\omega}}, \overline{\boldsymbol{\omega}})} \operatorname{Pr}\left(\psi_{i} \geq \underline{y}_{i}-\gamma\right) & \geq 1-\frac{\alpha}{2}, \\
\forall i & \in\{1, \ldots, N\} \\
\inf _{\boldsymbol{\omega} \sim \mathcal{P}(\boldsymbol{\mu}, \boldsymbol{\Sigma}, \underline{\boldsymbol{\omega}}, \overline{\boldsymbol{\omega}})} \operatorname{Pr}\left(\psi_{i} \leq \bar{y}_{i}+\gamma\right) & \geq 1-\frac{\alpha}{2}, \\
\forall i & \in\{1, \ldots, N\} .
\end{aligned}
$$

From the data-driven method described in [13], we can conservatively approximate (13) with the auxiliary decision variables, $\mathbf{Z} \in \mathbb{R}^{2 \times N}$; scalar parameters $\left(\kappa_{M}, \phi_{M}\right) ;$ and the following set of deterministic second-order cone constraints:

$$
\begin{aligned}
& \left(\left[\begin{array}{c}
\mathbf{y}_{\mathrm{ref}} \\
\widehat{\mathbf{p}} \\
\widehat{\mathbf{q}}
\end{array}\right]+\left[\begin{array}{c}
\mathbf{0} \\
\widehat{\boldsymbol{\mu}}_{M}
\end{array}\right]\right) \chi_{i}\left[\begin{array}{l}
\mathbf{1} \\
\mathbf{g}_{p} \\
\mathbf{g}_{q}
\end{array}\right] \\
& +\kappa_{M} \sqrt{\frac{1-\frac{\alpha}{2}}{\frac{\alpha}{2}}}\left\|\mathbf{Z}_{:, i}\right\|_{2}+\sqrt{\frac{\phi_{M}}{2}} Z_{2, i}-\gamma \leq \bar{y}_{i}, \\
& -\left(\left[\begin{array}{c}
\widehat{\mathbf{p}} \\
\widehat{\mathbf{q}}
\end{array}\right]+\left[\begin{array}{c}
\mathbf{0} \\
\widehat{\boldsymbol{\mu}}_{M}
\end{array}\right]\right)^{\top} \quad \boldsymbol{\chi}_{i}\left[\begin{array}{c}
\mathbf{1} \\
\mathbf{g}_{p} \\
\mathbf{g}_{q}
\end{array}\right] \\
& +\kappa_{M} \sqrt{\frac{1-\frac{\alpha}{2}}{\frac{\alpha}{2}}}\left\|\mathbf{Z}_{:, i}\right\|_{2}+\sqrt{\frac{\phi_{M}}{2}} Z_{2, i}-\gamma \leq-\underline{y}_{i},
\end{aligned}
$$

$$
\begin{aligned}
& \left\|\left[\begin{array}{cc}
\mathbf{0} & \mathbf{0} \\
\mathbf{0} & \widehat{\mathbf{\Sigma}}_{M}
\end{array}\right]^{\frac{1}{2}} \boldsymbol{\chi}_{i}\left[\begin{array}{c}
\mathbf{1} \\
\mathbf{g}_{p} \\
\mathbf{g}_{q}
\end{array}\right]\right\|_{2} \leq Z_{1, i}, \\
& \forall i \in\{1, \ldots, N\} \quad(14 \mathrm{c}) \\
& \sqrt{\frac{\phi_{M}}{2}}\left\|\left[\begin{array}{ll}
\mathbf{0} & \operatorname{diag}\left(\widehat{\overline{\boldsymbol{\omega}}}_{M}-\underline{\widehat{\boldsymbol{\omega}}}_{M}\right)
\end{array}\right] \boldsymbol{\chi}_{i}\left[\begin{array}{c}
\mathbf{1} \\
\mathbf{g}_{p} \\
\mathbf{g}_{q}
\end{array}\right]\right\|_{1} \leq Z_{2, i}, \\
& \forall i \in\{1, \ldots, N\} . \quad(14 \mathrm{c})
\end{aligned}
$$

The first term in (14a) and (14b) represents the average voltage magnitude deviation, whereas the second and third terms are tantamount to safety buffers to account for the variation in forecasting errors through $Z_{1, i}$ and (14c) and the estimation uncertainty of the sample moments through $Z_{2, i}$ and (14d).

The following theorem provides the guidelines on how to set the scalar parameters $\left(\kappa_{M}, \phi_{M}\right)$ so that the satisfaction of the data-driven deterministic constraints (14) implies the satisfaction of the distributionally robust chance constraints (13).

Theorem 1 ([13], Corollary 1). Assume that estimated supports bound the true supports, i.e., $\widehat{\widehat{\omega}}_{M} \leq \underline{\omega}$ and $\widehat{\hat{\boldsymbol{\omega}}}_{M} \geq \overline{\boldsymbol{\omega}}$, and that the number of independent and identically distributed samples, $M$, satisfies:

$$
8 \sqrt{\frac{M}{\exp \left((\sqrt{M}-2)^{2}\right)}}<\alpha
$$


If the scalar parameters in (14) are set to:

$$
\begin{aligned}
\kappa_{M} & =\sqrt{\frac{\sqrt{M}}{\sqrt{M}-1}}, \\
\phi_{M} & =\frac{2+\sqrt{2 \ln (8 \sqrt{M} / \alpha)}}{\sqrt{M}},
\end{aligned}
$$

then (14) is a conservative approximation to (13). And as $M \rightarrow \infty$, (14) asymptotically approaches (13).

The asymptotic result comes from the fact that as the number of samples, $M$, approaches infinity, then $\kappa_{M}$ approaches 1, $\phi_{M}$ approaches 0 , and the moments estimates approach their true values. At that point, (14) becomes the form found in [17], which was proven to be equivalent to (13).

Remark 2. If the estimated support vectors $\left(\underline{\widehat{\boldsymbol{\omega}}}_{M}, \widehat{\widehat{\boldsymbol{\omega}}}_{M}\right)$ do not completely bound the true support vectors $(\underline{\boldsymbol{\omega}}, \overline{\boldsymbol{\omega}})$ but instead bound them with probability $1-\epsilon$ for $\epsilon \epsilon$ $\left(0, \frac{\alpha}{2}\right)$, then Theorem 1 will remain true if $\alpha$ is replaced with $\widehat{\alpha}:=\frac{\alpha-2 \epsilon}{1-\epsilon}$. See Proposition 1 in [13] for more details.

Remark 3. The bound on the number of samples, $M$, imposed by (15) can be found numerically, but it is a mild constraint. For example, if $\alpha=0.01$, then $M$ would only need to be as large as 38 to satisfy the assumption.

For a system operator trying to minimize the probability of voltage magnitudes going outside of their bounds, it may be tempting to push the probability of violation $\alpha$ towards zero. However, a smaller $\alpha$ increases the size of the coefficients in front of the second and third LHS terms in Equations (14a) and (14b) and the coefficient in front of the LHS of Equation (14d). The increased size of $\phi_{M}$ due to a smaller $\alpha$ could be counteracted with a larger number of samples $M$ but the value of $\sqrt{\frac{1-\frac{\alpha}{2}}{\frac{\alpha}{2}}}$ will increase regardless. An increased size of the coefficients shrinks the feasible region for the droop coefficients. This could require a larger slack variable $\gamma$ that essentially expands out the bounds on the voltage magnitudes that are guaranteed for the specified probability of violation $\alpha$. In other words, lowering $\alpha$ does not come without cost to expanding the bounds on the voltage magnitude.

\subsection{Data-Driven Droop Control Scheme}

Finally, we summarize the complete voltage droop control scheme that uses the aforementioned data-driven, distributionally robust chance constraints for a given specified probability of violation, $\alpha$, and a number of collected forecast error samples, $M$.

At each droop control setting decision epoch (e.g., every $15 \mathrm{~min}$ ):

1. Collect the most recent $M$ power injection forecast errors $\left(\boldsymbol{\omega}^{p}, \boldsymbol{\omega}^{q}\right)$ from each node with nonzero power injections in the power network at the granularity of the droop control actions (e.g., every $100 \mathrm{~ms}$ ), and form the sample mean vector, $\widehat{\boldsymbol{\mu}}_{M}$; covariance matrix, $\widehat{\boldsymbol{\Sigma}}_{M}$; and support vectors, $\left(\underline{\widehat{\boldsymbol{\omega}}}_{M}, \widehat{\overline{\boldsymbol{\omega}}}_{M}\right)$.

2. Set $\left(\kappa_{M}, \phi_{M}\right)$ according to (16).

3. Solve the following optimization problem as a surrogate to problem (5):

$$
\begin{array}{rl}
\min _{\mathbf{g}_{p}, \mathbf{g}_{q}, \gamma, \beta, \mathbf{Z}} & f\left(\mathbf{g}_{p}, \mathbf{g}_{q}\right)+c_{\alpha} \gamma+c_{\beta} \beta \\
\text { s.t. } & (9),(14) \\
& \mathbf{g}_{p} \geq \mathbf{0}, \mathbf{g}_{q} \geq \mathbf{0}, \gamma \geq 0 \\
& \beta \leq \beta_{0}
\end{array}
$$

where $\beta_{0}<1$, and the cost coefficient, $c_{\beta}$, limits the size of the optimal solution as measured by the spectral radius, which was assumed before.

4. At each droop control action time step, operate the droop controller using the coefficients $\mathbf{g}_{p}, \mathbf{g}_{q}$, where power injections follow the expression (3).

Although the development of this scheme was based on the assumption of the linear power flow equations and the forecast errors that are independent and identically distributed, in the next section we test the scheme with nonlinear three-phase power flow and real-world power injection data.

\section{Performance Evaluation}

In this section, we evaluate the performance of the proposed data-driven voltage droop control setting scheme summarized in Section 3.3. For this purpose, we use a three-phase radial distribution network. The proposed approach is compared to a standard method of setting the droop control coefficients [3].

\subsection{Setup}

The unbalanced, three-phase, delta-connected IEEE 37-node distribution test case [20] was used to benchmark the performance of the droop control schemes. We assume that PV systems are installed on buses $5,6,7,9,10,11,13,14,16,20,21,24,26,29$, 


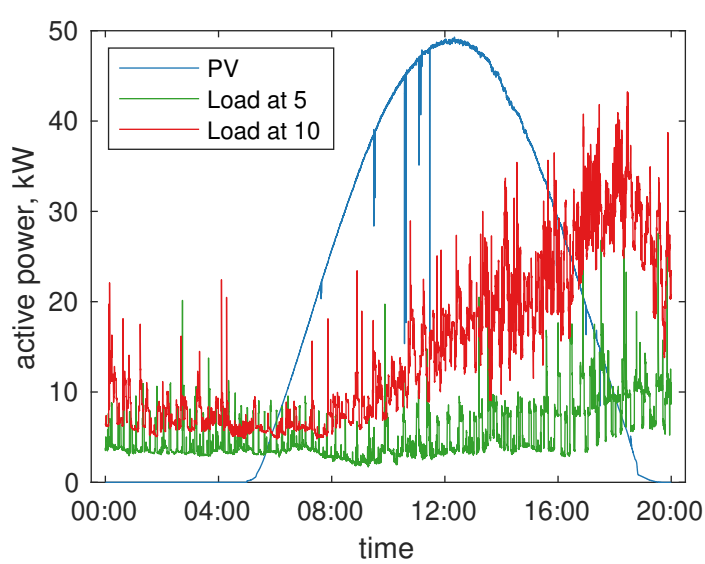

Figure 1: A sample of the PV and active load data at nodes 5 and 10 from [19].

$30,32,33,35$, and 36 . In addition, the input 1 -second (active) load and PV data were taken from feeders near Sacramento, California, on August 1, 2012, from 12 a.m.-8 p.m., which was a clear day [19]. See Figure 1 for a small sample of the data. The reactive power was set to be $50 \%$ of the magnitude of the active power load data to give a constant power factor of 0.894 for the uncontrollable loads. The power injections input data were scaled up by a factor of 15 to induce voltage violations under the scenario with no droop control. The targeted voltage magnitude lower and upper bounds were set to 0.95 and 1.05 p.u., respectively. To assess the performance of the droop control schemes on a realistic setup, the tuning of the schemes was done using the linearized power flow model, but their power injection control actions in (3) were tested with voltage magnitude measurements based on the nonlinear AC power flow model. The nonlinear power flow equations were solved on MATLAB using a three-phase fixed-point solver [21].

At each node and phase with a load, an inverter with a rating of $500 \mathrm{kVA}$ and a power source was added. The maximum voltage range that an inverter can react was set to be 0.1 p.u. from $v_{\text {ref }}=1$ p.u. which together with the rating constrains the capacity on each node with a droop controller as follows:

$$
\left\|\left[\begin{array}{ll}
g_{p, i} & g_{q, i}
\end{array}\right]^{\top}\right\|_{2} \leq 5000 .
$$

The linearization method to generate $(\mathbf{R}, \mathbf{B}, \mathbf{a})$ came from [14], which linearizes the power flow equations around the zero-load voltage. For the proposed data-driven, chance-constrained droop control, the droop control settings are recalculated every 15 minutes using the most recent forecast errors. The default amount of the sampled forecast error data used to calculate the sample moments is the previous 3 hours.

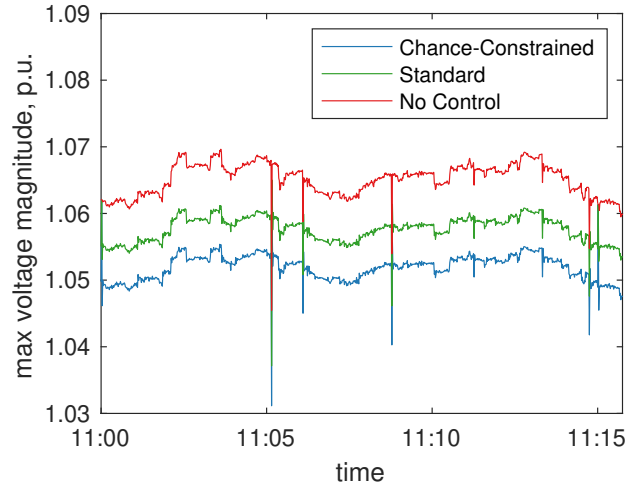

(a) Maximum voltage magnitudes

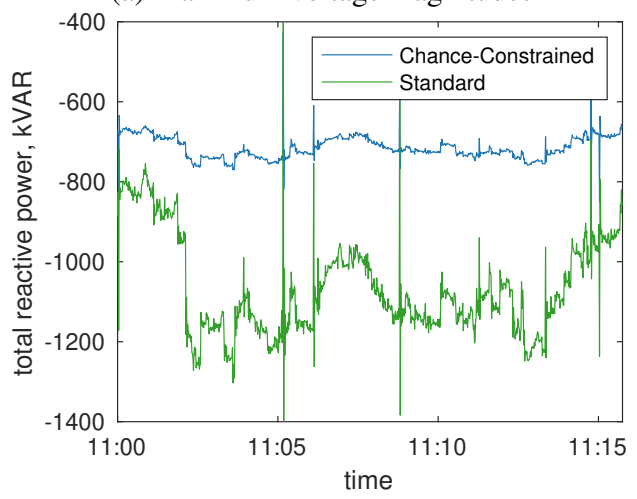

(b) Total reactive power

Figure 2: (a) Maximum voltage magnitudes, and (b) total reactive power injected from control from 11:00 a.m.-11:15 a.m. under different control schemes.

That is, the chance constraints of a control period are calculated based on the forecast errors collected in the previous 3-hour period. Later in this section, we will vary this duration to analyze the effect of increasing the amount of data samples on the performance of the proposed approach. The forecasting method used was the persistent estimator algorithm, i.e., the forecast $(\widehat{\mathbf{p}}, \widehat{\mathbf{q}})$ for the next 15 minutes is the average uncontrolled power injections from the previous 15 minutes. The allowable probability of violation, $\alpha$, for the chance constraints is set to 0.1. The maximum allowable bound, $\beta_{0}$, on (9) is set to 0.95 . The objective function was set with $f\left(\mathbf{g}_{p}, \mathbf{g}_{q}\right)=\left\|\left[\mathbf{g}_{p} \mathbf{g}_{q}\right]^{\top}\right\|_{\mathrm{F}}^{2}$ to promote a wide distribution of control, and the cost coefficients $c_{\alpha}=5 \times 10^{7}$ and $c_{\beta}=10^{3}$.

As a benchmark comparison against the proposed data-driven method, we employ the IEEE 1547 guidelines [3] (Figure 2a) as the standard way to set the voltage droop controls, which include a deadband between 0.98 and 1.02 p.u. and a limit of 0.8 on the maximum fraction of the apparent power that can be used by any inverter for reactive power. 


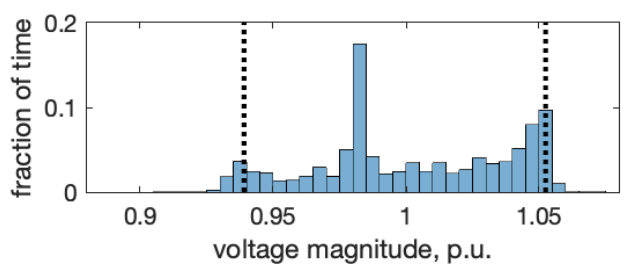

(a) Chance-constrained droop control

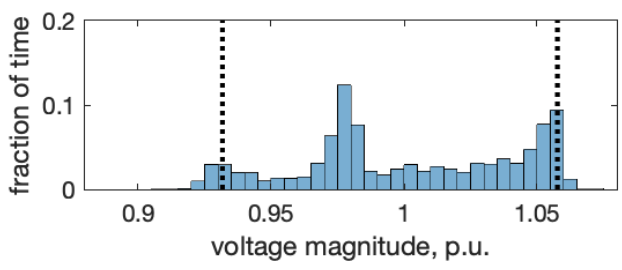

(b) Standard droop control settings

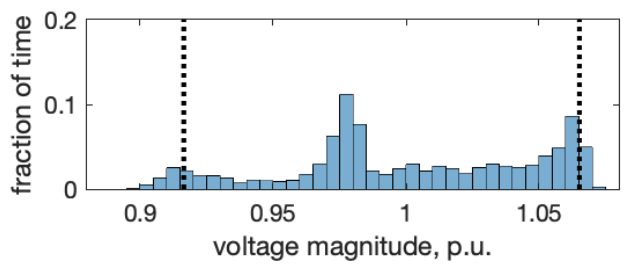

(c) No droop control

Figure 3: Histograms of voltage magnitude at Node 32 (Phase a) under different control schemes. Outside the dotted lines represents the tails, which contain $5 \%$ of the occurrences on each side.

\subsection{Results}

First, we compare the performance between the data-driven, chanced-constrained droop control setting scheme and the standard control settings that are blind to the power injection data and power system properties. During the 15-minute interval from 11:00 a.m. to 11:15 a.m., the uncontrolled system had its peak voltage magnitude among all nodes and phases as a result of the high penetration of solar power. Figure $2 \mathrm{a}$ tracks the maximum voltage in the system during this interval under the considered control schemes. Although the standard control settings can reduce the maximum voltage magnitude from the uncontrolled system by 0.0075 p.u. on average, the proposed chance-constrained scheme can reduce it by 0.0133 p.u. and keep it close to the 1.05 p.u. upper bound. Figure $2 b$ depicts the total reactive power being injected among all the droop controllers in the system. This demonstrates the efficacy of the proposed chance-constrained scheme with respect to using power resources for voltage control because it is using 33\% less reactive power than the standard settings, yet it can provide substantial reduction of the maximum voltage.

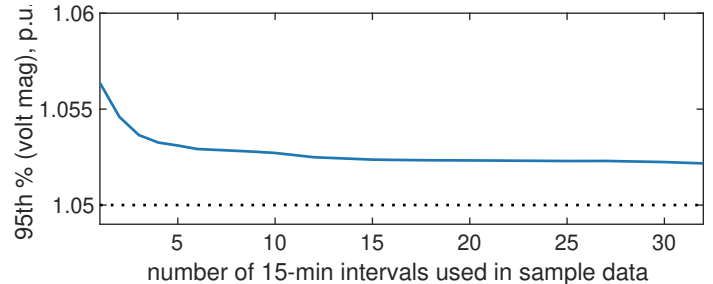

(a) 95th percentile of voltage magnitude

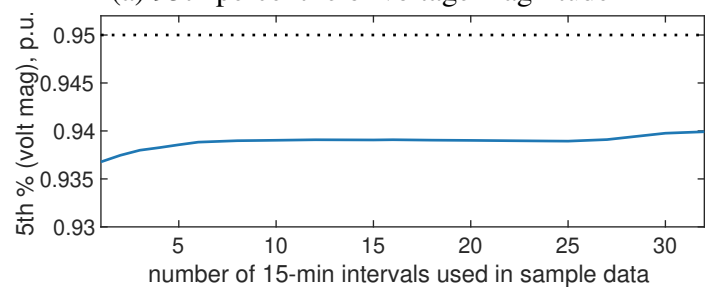

(b) 5th percentile of voltage magnitude

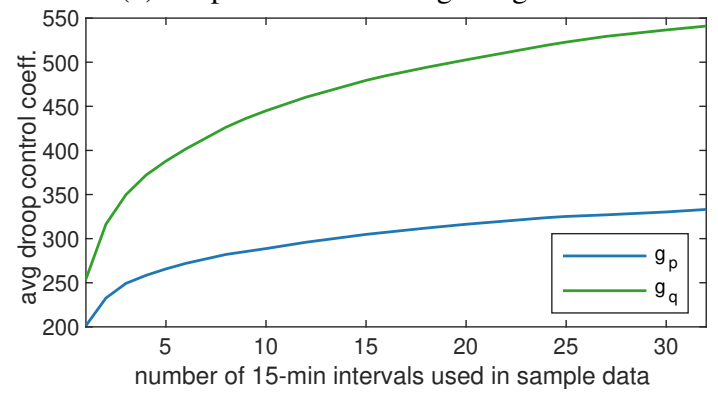

(c) Average droop control coefficients

Figure 4: (a) 95th and (b) 5th percentile of the voltage magnitudes at Node 32 (Phase a) and (c) average droop control coefficients versus the number of 15-minute intervals used in the sample data.

To assess the performance over the whole 20 hour period, we showcase the voltage magnitude of a particularly stressed node. Node 32 (Phase a), located at the edge of the network, achieves both the minimum and maximum voltage magnitude throughout the whole period for the uncontrolled scenario. Figure 3 shows the histograms of its voltage magnitude over the considered 20-hour period under the different control schemes. The dotted lines delineate the $\frac{\alpha}{2}=0.05$ tails on either side, within which the chance-constrained scheme was aiming to place the 0.95-p.u. and 1.05-p.u. bounds. Although the standard control was able to squeeze the voltage magnitudes toward the 1.00-p.u. reference voltage magnitude, the data-driven, chance-constrained scheme was able to do it more effectively. More importantly, the proposed approach was almost able to meet the requirement of the chance constraints to get the lower and upper tails to start at the 0.95-p.u. and 1.05 -p.u. bounds, respectively.

One assumption made in the development of the 


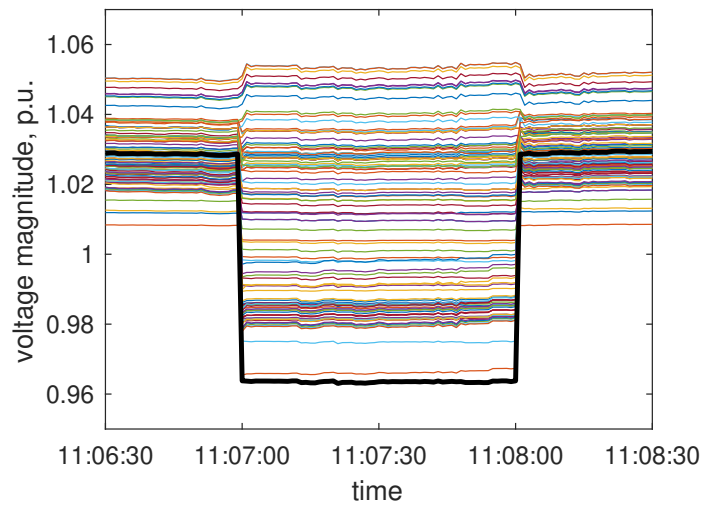

Figure 5: Voltage magnitudes at the buses and their phases during a fault at Bus 16 (Phase b) under the data-driven, chance-constrained droop control scheme. The thick black line indicates the voltage magnitudes at the location of the fault.

data-driven, chance-constrained droop control scheme was that the forecasting errors were independent and identically distributed. In this case, the more historical samples that are used to find the mean and covariance, the more accurate the sample moments will be; however, if the probability distribution of the forecasting errors varies in time, more samples from farther back in time could lead to inaccurate sample moments. In Figure 4, we varied the number of samples used in calculating the sample mean and covariance in terms of the number of past 15-minute intervals. We again focused on the voltage magnitude of Node 32 (Phase a) and looked at the 95th and 5th percentiles in Figures $4 \mathrm{a}$ and $4 \mathrm{~b}$, respectively. With $\alpha=0.1$, these are the percentiles focused on by the chance constraints. As the number of samples increases, the percentiles get closer to the desired bounds of 0.95 and 1.05 p.u., but they asymptotically approach a gap that separates them. On the other hand, Figure 4c shows the droop coefficients averaged over time and the node location, which grow logarithmically with the number of samples. This is mainly because of the chance-constraint parameters $\left(\kappa_{M}, \phi_{M}\right)$ approaching $(1,0)$, which allows for larger feasible droop control coefficients. Larger droop control coefficients, however, result in a more aggressive usage of the energy resources; thus, when picking the number of past samples to use for estimating the sample moments, a practitioner might balance the improvement to the 5th and 95th percentile voltage bounds to the increase in droop control coefficients.

Another assumption made during the development of the droop control scheme was that the droop control actions act fast enough so that changes in the uncontrollable load would be relatively small compared to the magnitude of the uncontrollable loads themselves. Although this is typically true, we want to test how the droop controls behave when at an instance when this is not true (e.g., under a fault). Thus, we simulated a fault at Bus 16 (Phase b) by increasing the active load by twice the amount of the total average load (excluding the renewable generation) in the system at 11:07 am and then releasing it at 11:08 am. Figure 5 shows the voltage magnitudes at each bus and phase during this time interval. Notice that the system has large changes in voltage at the beginning and end of the time interval for the simulated fault, but it does not show any signs of instability.

\section{Conclusion}

In this paper, we develop a data-driven method to set voltage droop control coefficients in an electrical distribution network with high integration levels of DERs. The main objective is to limit the probability of voltage magnitudes going outside their bounds. The method is based on a chance-constrained stochastic optimization problem and adapts the method developed by [13] to conservatively approximate the chance constraints with the sample mean and covariance derived from historical data. The proposed method is evaluated on an unbalanced, three-phase, delta-connected IEEE 37-node distribution test case [20]. We show that it can more effectively keep the voltage magnitudes within their prescribed bounds than the IEEE 1547 guidelines [3]. Future directions include analyzing the stability of networked DERs with droop controls based on the nonlinear power flow model and studying the design of voltage droop control under various objectives, such as determining the minimum number of DERs with droop control that are required to maintain the voltage magnitudes between bounds. It will also be interesting to explore the tradeoff between the number of samples and the lowest attainable probability of violation for the bounds on the voltage magnitudes.

\section{Acknowledgments}

This work was authored by the National Renewable Energy Laboratory (NREL), operated by Alliance for Sustainable Energy, LLC, for the U.S. Department of Energy (DOE) under Contract No. DE-AC36-08GO28308. This work was supported by the Laboratory Directed Research and Development (LDRD) Program at NREL. The views expressed in the article do not necessarily represent the views of the DOE or the U.S. Government. The U.S. Government retains and the publisher, by accepting the article for 
publication, acknowledges that the U.S. Government retains a nonexclusive, paid-up, irrevocable, worldwide license to publish or reproduce the published form of this work, or allow others to do so, for U.S. Government purposes.

\section{References}

[1] E. J. Coster, J. M. Myrzik, B. Kruimer, and W. L. Kling, "Integration issues of distributed generation in distribution grids," Proceedings of the IEEE, vol. 99, no. 1 , pp. 28-39, 2010.

[2] T. Verschueren, K. Mets, B. Meersman, M. Strobbe, C. Develder, and L. Vandevelde, "Assessment and mitigation of voltage violations by solar panels in a residential distribution grid," in 2011 IEEE International Conference on Smart Grid Communications (SmartGridComm), pp. 540-545, IEEE, 2011.

[3] S. Chakraborty, A. Hoke, and B. Lundstrom, "Evaluation of multiple inverter volt-var control interactions with realistic grid impedances," in 2015 IEEE Power \& Energy Society General Meeting, pp. 1-5, IEEE, 2015.

[4] K. Baker, A. Bernstein, E. Dall'Anese, and C. Zhao, "Network-cognizant voltage droop control for distribution grids," IEEE Transactions on Power Systems, vol. 33, no. 2, pp. 2098-2108, 2017.

[5] A. Singhal, V. Ajjarapu, J. Fuller, and J. Hansen, "Real-time local volt/var control under external disturbances with high pv penetration," IEEE Transactions on Smart Grid, vol. 10, no. 4, pp. 3849-3859, 2018.

[6] R. A. Jabr, "Robust volt/var control with photovoltaics," IEEE Transactions on Power Systems, vol. 34, no. 3 , pp. 2401-2408, 2019.

[7] J. Von Appen, M. Braun, T. Stetz, K. Diwold, and D. Geibel, "Time in the sun: the challenge of high pv penetration in the german electric grid," IEEE Power and Energy magazine, vol. 11, no. 2, pp. 55-64, 2013.

[8] H. Sun, Q. Guo, J. Qi, V. Ajjarapu, R. Bravo, J. Chow, Z. Li, R. Moghe, E. Nasr-Azadani, U. Tamrakar, et al., "Review of challenges and research opportunities for voltage control in smart grids," IEEE Transactions on Power Systems, vol. 34, no. 4, pp. 2790-2801, 2019.

[9] C. M. Lagoa, X. Li, and M. Sznaier, "Application of probabilistically constrained linear programs to risk-adjusted controller design," in Proceedings of the 2001 American Control Conference.(Cat. No. 01CH37148), vol. 2, pp. 738-743, IEEE, 2001.

[10] A. Nemirovski and A. Shapiro, "Convex approximations of chance constrained programs," SIAM Journal on Optimization, vol. 17, no. 4, pp. 969-996, 2007.

[11] C. M. Lagoa, "On the convexity of probabilistically constrained linear programs," in Proceedings of the 38th IEEE Conference on Decision and Control (Cat. No. 99CH36304), vol. 1, pp. 516-521, IEEE, 1999.

[12] D. Bertsimas, V. Gupta, and N. Kallus, "Data-driven robust optimization," Mathematical Programming, vol. 167, no. 2, pp. 235-292, 2018.

[13] J. Comden, A. S. Zamzam, and A. Bernstein, "Data-driven moment-based distributionally robust chance-constrained optimization," arXiv preprint arXiv:2109.08742, 2021.
[14] A. Bernstein and E. Dall'Anese, "Linear power-flow models in multiphase distribution networks," in 2017 IEEE PES Innovative Smart Grid Technologies Conference Europe (ISGT-Europe), pp. 1-6, IEEE, 2017.

[15] Y. Liu, N. Zhang, Y. Wang, J. Yang, and C. Kang, "Data-driven power flow linearization: A regression approach," IEEE Transactions on Smart Grid, vol. 10, no. 3, pp. 2569-2580, 2018.

[16] R. J.-B. Wets, "Chapter viii stochastic programming," Handbooks in operations research and management science, vol. 1, pp. 573-629, 1989.

[17] L. E. Ghaoui, M. Oks, and F. Oustry, "Worst-case value-at-risk and robust portfolio optimization: A conic programming approach," Operations research, vol. 51, no. 4, pp. 543-556, 2003.

[18] G. A. Hanasusanto, V. Roitch, D. Kuhn, and W. Wiesemann, "A distributionally robust perspective on uncertainty quantification and chance constrained programming," Mathematical Programming, vol. 151, no. 1, pp. 35-62, 2015.

[19] J. Bank and J. Hambrick, "Development of a high resolution, real time, distribution-level metering system and associated visualization, modeling, and data analysis functions," tech. rep., National Renewable Energy Lab.(NREL), Golden, CO (United States), 2013.

[20] "IEEE 37 Node Test Feeder," tech. rep., IEEE, Power Engineering Society, Distribution System Analysis Subcommittee, 1992. https://site.ieee.org/pes-testfeeders/resources/.

[21] A. Bernstein, C. Wang, E. Dall'Anese, J.-Y. Le Boudec, and C. Zhao, "Load flow in multiphase distribution networks: Existence, uniqueness, non-singularity and linear models," IEEE Transactions on Power Systems, vol. 33, no. 6, pp. 5832-5843, 2018. 